\title{
Martin Kirnbauer
}

\section{»sind alle lang« - Glareans Erläuterungen zur Mensural- notation und musikalische Praxis}

1516 erschien in Rom in der Offizin von Andrea Antico ein Folio-Chorbuch mit dem Titel Liber qvindecim missarvm electarvm qvae per excellentissimos mosicos compositae foervnt (RISM 1516 ${ }^{1}$ ). ${ }^{1}$ Bereits 1513 hatte Antico durch ein päpstliches Privileg zu erreichen versucht, an dem in Italien von Ottaviano Petrucci beherrschten Markt für gedruckte Musikalien teilzuhaben. Ein zweites Privileg vom 27. Januar 1516 galt für einen Druck »in magno volumine «, jenem bald darauf am 9. Mai desselben Jahres erscheinenden Liber quindecim missarum mit Messen von Antoine Brumel, Jean Mouton, Antoine de Fevin, Pierre de la Rue, einem gewissen "petrus roselli« und von Josquin Desprez. Von den seinerzeit insgesamt etwa 1000 gedruckten Exemplaren weist RISM heute noch dreizehn Kopien nach, darunter eines in der Stadtbibliothek Baden in der Schweiz, zwischen Basel und Zürich gelegen. ${ }^{2}$ Dieser Band stammt ursprünglich aus dem Badener Chorherrenstift, das 1624 gegründet und 1875 aufgehoben wurde, wobei die Bibliotheksbestände in die Vorgängerinstitution der heutigen Stadtbibliothek gelangten. ${ }^{3}$ Er weist kaum Gebrauchsspuren auf, mit Ausnahme allerdings von einigen teils bemerkenswerten handschriftlichen Einträgen. ${ }^{4}$ Diese finden sich fast ausschließlich in der zehnten Messe, Josquins Missa de beata virgine. Am spektakulärsten ist wohl der Beginn (fol. $\mathrm{Cxiv}^{v}-\mathrm{cxv}^{r}$, siehe Abbildung 1). Neben einer griechischen Zeile oben links findet sich auf der rechten Seite zwi-

1 Zum Folgenden siehe Iain Fenlon, Music, Print and Culture in Early Sixteenth-Century Italy, London 1995 (The Panizzi Lectures, 1994), insbes. S. 28-34.

2 RISM B I 1: Recueils imprimés XVIe-XVIIe siècles, hrsg. von François Lesure, München 1960 (Répertoire International des Sources Musicales, B.1.1), S. 97, allerdings mit einer irreführenden Sigel-Angabe, da sich der Band nicht im Historischen Museum (CH-BA), sondern in der Stadtbibliothek befindet; Signatur »Stift Nr. $21 \ll .-$ Demnächst wird ein Faksimile dieses Drucks im Cornetto-Verlag (Stuttgart) erscheinen, von dem auch die Vorlagen für die Abbildungen freundlicherweise zur Verfügung gestellt wurden.

3 Siehe Otto Mittler, Geschichte der Stadt Baden, Bd. 1: Von der frühesten Zeit bis um 1650, Aarau 1962, S. 342-348; Fritz Stalder und Adolf Rohr, "Zur Geschichte der Stadtbibliothek Baden«, in: Badener Neujahrsblätter 45 (1970), S. 82; Adolf Rohr, "Geschichte der Stadtbibliothek Baden«, in: Baden in Gedichten und Liedern aus 6 Jahrhunderten, hrsg. von Uli Münzel, Baden 1987, S. 290.

4 Ein Verzeichnis findet sich im Anhang. 


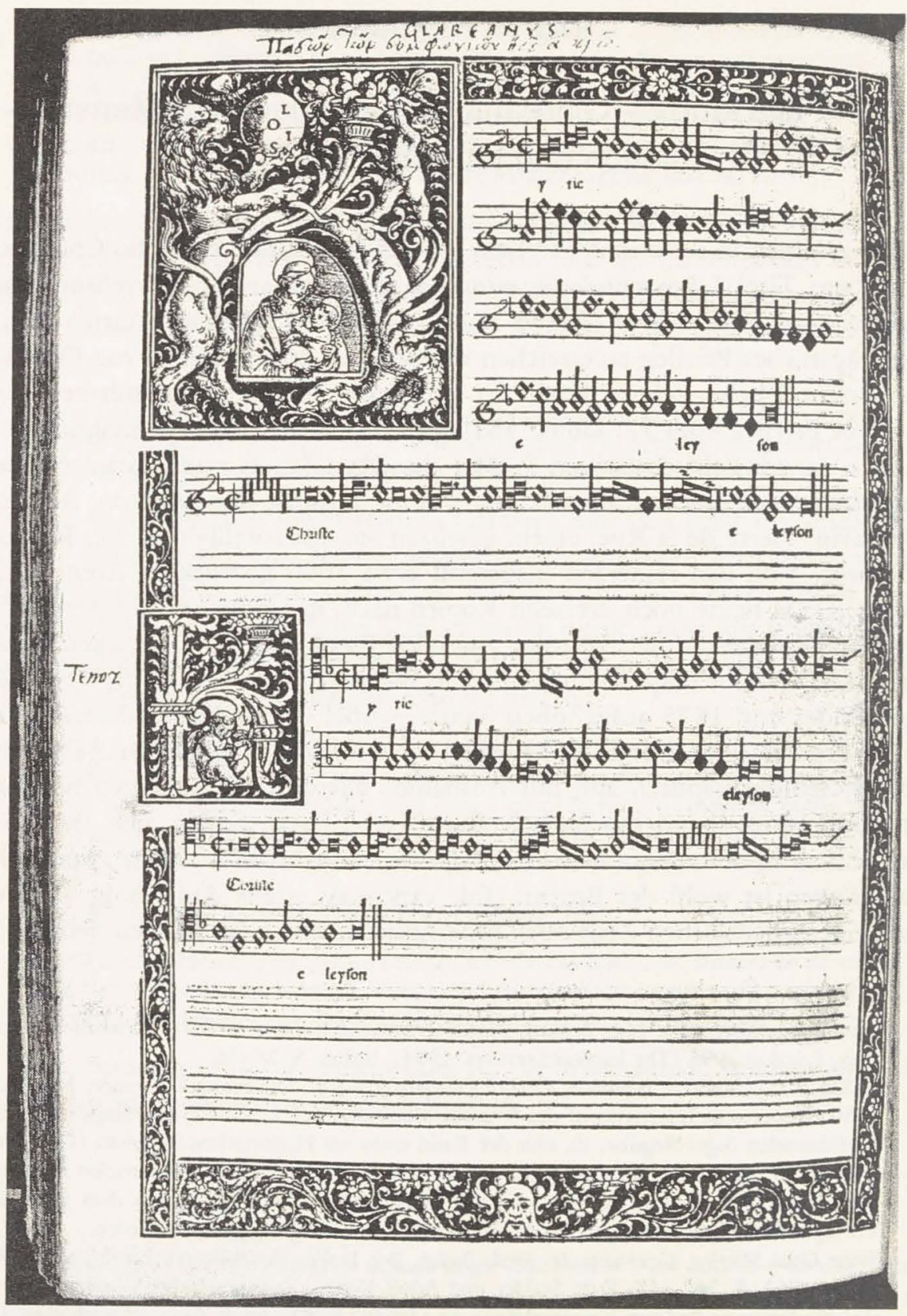

Abbildung 1: Liber quindecim missarum, Rom: Andrea Antico 1516, Exemplar 


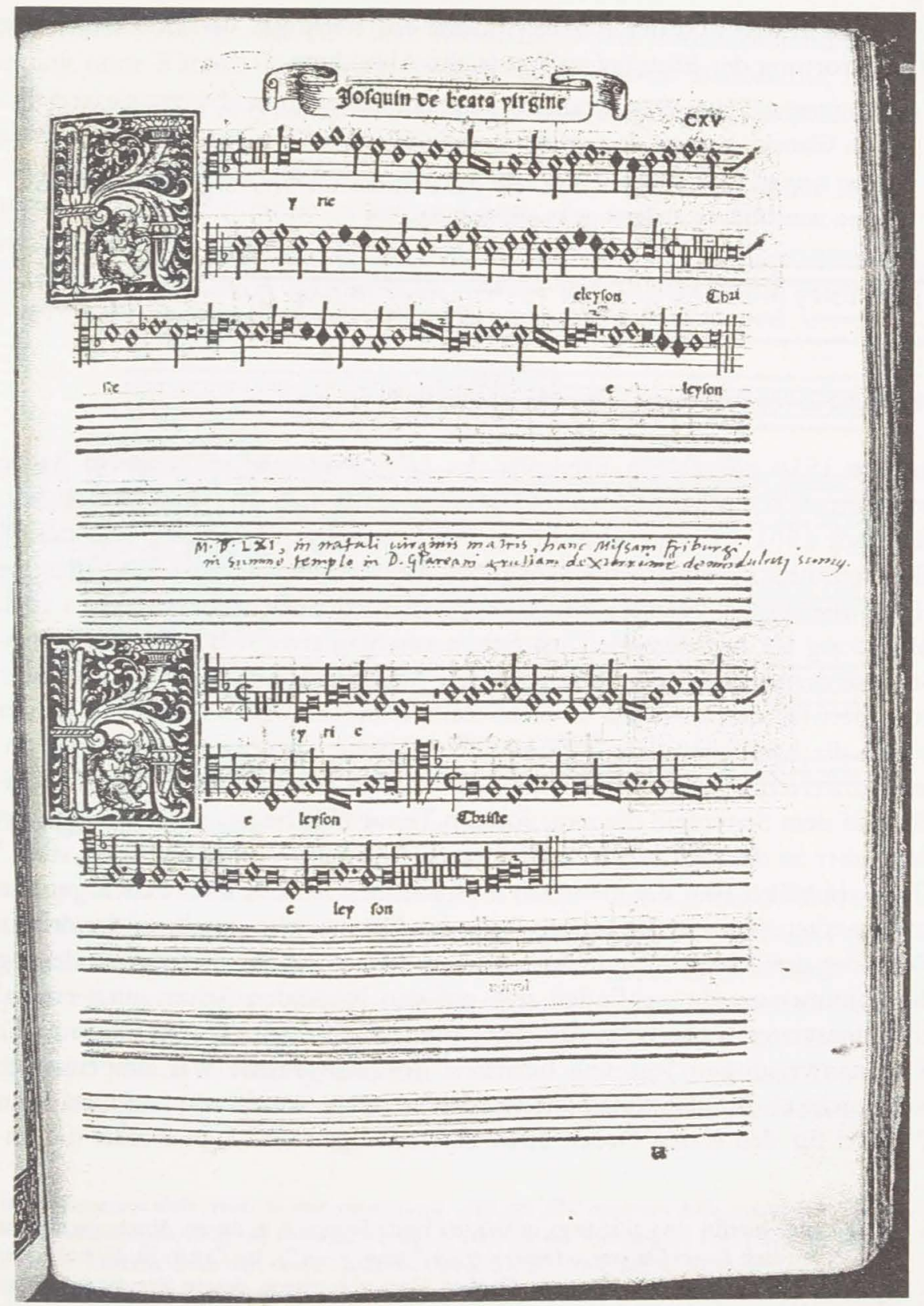

der Stadtbibliothek Baden, Schweiz, Sign. Stift Nr. 21, fol. $114^{\vee}$ und $115^{r}$ 
schen der Altus- und der Bassus-Stimme ein Vermerk, der eine Datierung und Verortung der Einträge zulässt (siehe Abbildung 2):

M.D.LXI, in natali uirginis matris, hanc Missam Friburgi in summo templo in D. Glareani gratiam dexterrime demodularj sumus.

1561, am 8. September, haben wir diese Messe im Freiburger Münster zu Ehren von Glarean äußerst gewandt gesungen.

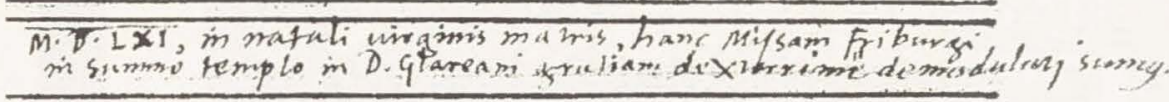

Abbildung 2: wie Abbildung 1, fol. $115^{r}$ (Ausschnitt)

In dem 1516 gedruckten Exemplar des Liber quindecim missarum in Baden liegt demnach ein Musikdruck mit Messen des frühen 16. Jahrhunderts vor, der noch $1561 \mathrm{zu}$ einer Aufführung der Musik Josquins diente und der zu diesem Zweck - von einem Cantor etwa - eingerichtet wurde, denn dieselbe Hand, die diesen Vermerk anbrachte, hinterließ noch weitere Spuren (vgl. Abbildung 1): Auf dem gleichen Seitenaufschlag etwa wurde in der BassusStimme der vom Drucker falsch gesetzte Schlüssel um eine Terz nach unten korrigiert (in der Mitte der zweiten Zeile zum Textwort »Christe«). Weiter wurde die handschriftliche Stimmenbezeichnung »Tenor« vor der links unten platzierten Stimme angebracht. Dies bezieht sich darauf, dass diese Position auf dem Seitenfeld die zwar für den Tenor übliche ist, auf der folgenden Seite aber an der gleichen Stelle der handschriftliche Vermerk »Altus « steht. ${ }^{5}$ Und schließlich sind die mehrfach zu findenden Ziffern über den Ligaturen zu bemerken (etwa in der letzten Zeile des Cantus oder jeweils in der dritten Zeile der drei übrigen Stimmen). Neben diesen Ziffern und der Änderung der Stimmenzuordnung finden sich auf den folgenden Seiten auch einmal ein zugesetztes Sicherheits-Akzidenz sowie Kommentare, die sich auf die Kanonanweisungen Josquins beziehen. Josquins Messe war ursprünglich wohl nicht als Einheit konzipiert worden, sondern wurde erst von Ottaviano Petrucci für den ersten Druck 1514 zusammengestellt. ${ }^{6}$ Dies erklärt die un-

5 Die Gründe hierfür sind allerdings unklar, da beide Stimmen in diesen Abschnitten einen fast identischen Stimmumfang aufweisen $(f-b$ " bzw. $g-b$ "). Im Credo ist die höher gehende Partie (ursprünglich Tenor $a-f^{\prime}$ ) dem Altus zugewiesen, dessen Stimme im Ambitus $c-e$ ' hingegen dem Tenor.

6 Siche Richard Sherr, "Notes on Two Roman Manuscripts of the Early Sixteenth Century«, in: Musical Quarterly 63 (1977), S. 48-73; ders., "The Relationship between a Vatican Copy of the Gloria of Josquin's Missa de Beata Virgine and Petrucci's Print«, in: Trasmissi- 
terschiedliche Anlage der einzelnen Messteile: Kyrie und Gloria sind vierstimmig ohne Kanon, die übrigen Teile (Credo, Sanctus, Benedictus, Agnus Dei) weisen unterschiedliche Kanons auf, aus denen sich eine fünfte Stimme ergibt.

Während der erste Satz mit einem Kanon, das Credo mit der gedruckten Kanonbeischrift »le premier va devant. «bzw. "Le devant va derriere." nicht weiter kommentiert wurde, ${ }^{7}$ finden sich handschriftliche Zusätze erstmals im Sanctus (fol. cxxiii'; siehe Abbildung 3). Hier wurde der gedruckte französisch-

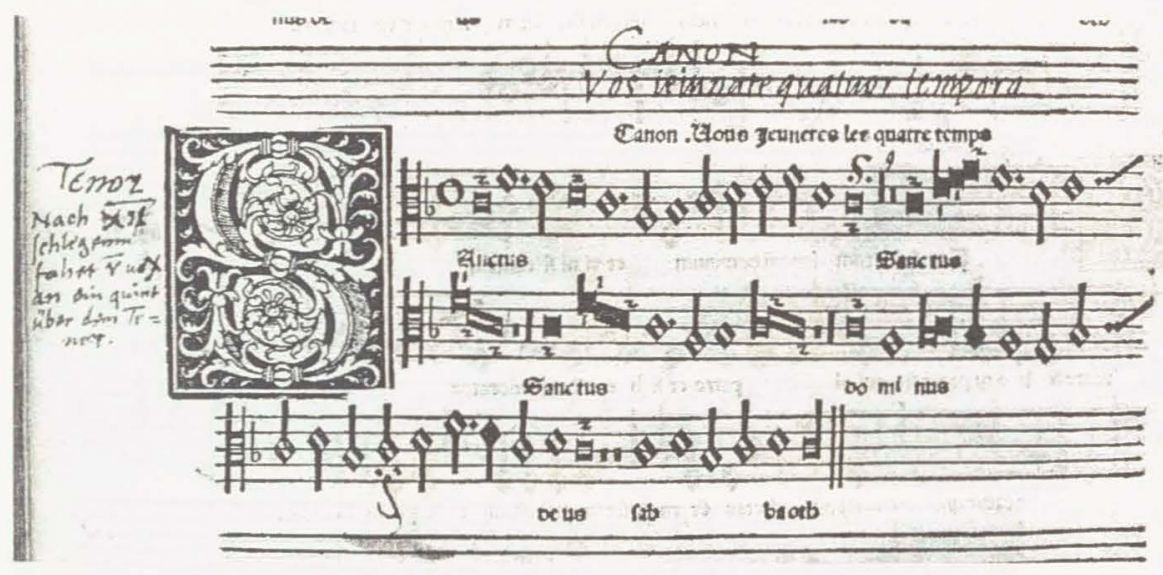

Abbildung 3: wie Abbildung 1, fol. $123^{v}$ (Ausschnitt)

sprachige »Canon. Uous jeuneres les quatre temps« auf Lateinisch übersetzt ("CANON / Vos ieiunate quatuor tempora «). Dies erschien vielleicht auch nicht allzu erhellend - Josquin meinte, dass eine fünfte Stimme nach vier tempora einsetzen sollte, dabei (unausgesprochen) die Tenorstimme eine Quinte höher wiederholend -, jedenfalls ist marginal zusätzlich auf Deutsch angemerkt: "Nach XII schlegenn fahrt V uox an ein quint über dem Tenor.« Etwas Ähnliches wiederholt sich auf der nächsten Seite (siehe Abbildung 4),

one e recenzione delle forme di cultura musicale. Atti del XIV congresso della società internazionale di musicologia, Bologna, Ferrara, Parma ... 1987, hrsg. von Angelo Pompilio, Donatella Restani, Lorenzo Bianconi und F. Alberto Gallo, Turin 1990, Bd. 2, S. 267.

7 In Anbetracht der expliziten Einträge in den anderen Kanon-Abschnitten könnte die Frage gestellt werden, ob dieses Credo 1561 vielleicht nur vierstimmig aufgeführt wurde. Allerdings erweist sich die kanonische Stimme mehrfach als struktureller, eigentlicher Tenor; siche Alejandro Enrique Planchart, "Masses on Plainsong Cantus Firmi «, in: The Josquin Companion, hrsg. von Richard Sherr, Oxford 2000, S. 124. 
hier lautet die marginale Notiz: »Quinta uox gath uß dem Tenor nach acht

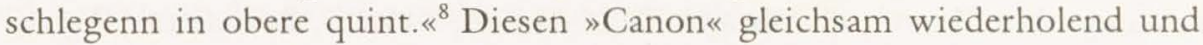
verdeutlichend steht im Druck ein Signum congruentiae für den Ort des Einsatzes der Quinta vox (erste Zeile). Der Bearbeiter von 1561 markierte zusätzlich mit einem gleichen Zeichen das Schließen dieser Stimme (letzte Zeile). Befolgt man diese Fermate, so enden Tenor und Quinta vox mit einem gemeinsam ausgehaltenen Schlusston (Quinte $c-g$ ), obwohl Josquin etwas raffinierter ein Weiterführen der kanonischen Stimme beabsichtigte, gemeinsam mit dem Cantus, Altus und Bassus schließend. ${ }^{9}$

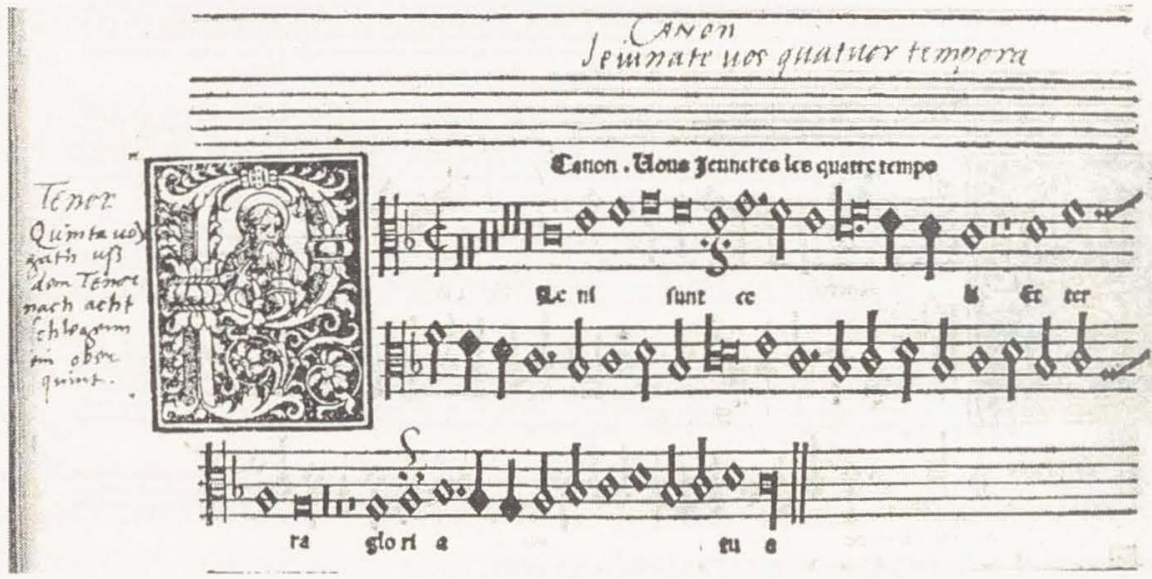

Abbildung 4: wie Abbildung 1, fol. $124^{\vee}$ (Ausschnitt)

Bemerkenswert an diesen »Canon«-Einträgen ist nicht die Übersetzung einer französischen Anweisung in ein mit lateinischsprachigen Fachbegriffen durchsetztes Deutsch - an anderer, ähnlicher Stelle findet sich zusätzlich » $\Delta \iota \alpha \pi \varepsilon v \tau \varepsilon \ll$ in griechischen Buchstaben $-^{10}$, sondern die unterschiedliche Übertragung der »quatre temps«: Im Sanctus, das im Tempus perfectum notiert ist, sind die vier Tempora zwölf »schlege«, im Abschnitt des im Tempus imperfectum diminutum notierten Pleni sunt ergeben sie acht.

8 Die Formulierung "gath uß dem Tenor nach acht schlegenn « könnte vielleicht sogar als Hinweis darauf interpretiert werden, dass der Sänger der Quinta vox zunächst den Tenor mitsingt und dann mit seiner Stimme einsetzt.

9 Gleiches findet sich im Abschnitt Pleni sunt und im Agnus Dei, nicht aber im Hosanna und Benedictus.

10 Benedictus (fol. cxxviv) marginal »Quinta uox ex Tenore post quatuor tempora in $\Delta 1 \alpha \pi \varepsilon v-$ $\tau \varepsilon \ll$; Agnus dei (fol. cxxviii ${ }^{v}$ ) marginal »Quinta uox ex Tenore post octo pausas in $\Delta t \alpha \pi \varepsilon v \tau \varepsilon \ll$. 
Nun gehört diese eigentümliche Verrechnung in »schlegen« in dieselbe Kategorie wie die oben genannten Ziffern über Ligaturen, welche die Längen der Notenwerte - und an anderer Stelle auch Pausen - auflösen. Dies sei anhand einiger Beispiele (siehe Tabelle) verdeutlicht:

a)
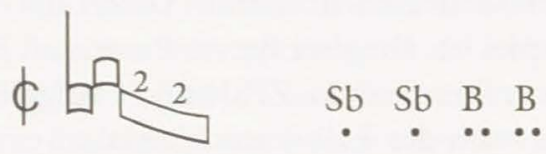

b) $\phi \stackrel{3}{\square}$

B

9

$\overline{\text { Fr }}$

c)

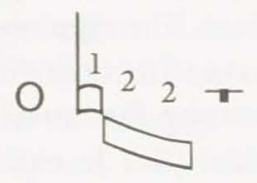

$\begin{array}{ccc}\mathrm{Sb} & \text { alt. Sb } & \mathrm{B} \\ - & \cdots & \end{array}$

$2^{2} 2^{2}$

d)

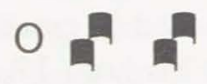

B B $B$ B

3

B

8

e) $\$ \square$

B: Brevis Sb: Semibrevis alt. Sb: alterierte Semibrevis Punkte bezeichnen die Anzahl der »schleg «

Tabelle: Beispiele für die Verrechnung von Notenwerten

Gleich auf der ersten Seite findet sich eine Ligatur cum opposita proprietate (beginnend mit zwei Semibreven), deren zweite Hälfte (mit zwei Breven) 
mit den Ziffern » 2 « überschrieben ist (siehe Beispiel a). Angegeben ist also, dass die beiden restlichen Töne der Ligatur jeweils zwei »schlege« dauern sollen.

Im »Cum sancto spiritus «, dem ersten dreizeitigen Abschnitt dieser Messe im Tempus perfectum diminutum, verdeutlicht eine Ziffer $» 3$ « über einer hier perfekten Brevis ihre Dauer von drei »schlegen«, während die Breven in den vorangehenden, in imperfekten Mensuren notierten Teilen der Messe nur jeweils zwei dauerten (siehe Beispiel b). Die drei Brevis-Pausen zu Beginn des Basses sind durch die Ziffer "9 « in ihre Gesamt-Zähleinheit aufgelöst.

Auf gleiche Weise ist im Sanctus auch der Fall einer Alteration einer Semibrevis angegeben (siehe Beispiel c).

An einer anderen Stelle wird die Imperfizierung einer kolorierten BrevisBrevis-Ligatur, also das Umschlagen von Drei- zu Zweizeitigkeit verdeutlicht, folgerichtig trägt die nächste weiße Brevis eine Ziffer »3 « (siehe Beispiel d).

Ein letztes Beispiel für diese sschlagende` Art der Verdeutlichung: Im zweistimmigen Agnus Dei gibt die Ziffer »8« über einer Maxima an, dass der Sänger hier 8 »schlege« bis zu seinem Einsatz zählen muss (siehe Beispiel e).

Zusammengefasst lässt sich festhalten, dass alle diese Einträge auf eine praktische Einrichtung der Josquin-Messe dieses Druckes für eine Aufführung hinweisen. Allerdings berücksichtigt diese Einrichtung fast ausschließlich elementare Aspekte, wie gerade das Verrechnen der hier ja eigentlich unkompliziert zu lesenden Ligatur- und Pausenwerte in Semibreven bzw. in sozusagen handfeste »schlege« zeigt.

Solche Ziffern zur Verdeutlichung von Notenwerten sind in einer Vielzahl von weiteren Quellen belegt, von der Mitte des 14. bis zum 17. Jahrhundert, in unterschiedlichen Zusammenhängen. ${ }^{11}$ Neben instrumentalen Kontexten finden sie sich oftmals in didaktischen Zusammenhängen bzw. konkreten Lehrsituationen, in denen sie die offenbar großen Schwierigkeiten

11 Vgl. etwa die Ziffern über einem Motetten-Tenor von Johannes de Muris, siehe Lawrence Gushee, »New Sources for the Biography of Johannes de Muris «, in: Journal of the American Musicological Society 22 (1969), S. 3-26, Plate II; mit falscher Angabe der Quellensignatur auch abgebildet in Die Musik des Mittelalters, hrsg. von Hartmut Möller und Rudolf Stephan, Laaber 1991 (Neues Handbuch der Musikwissenschaft, 2), S. 354; handschriftliche Ergänzung in einem Stimmbuch eines Canzonen-Drucks von Girolamo Frescobaldi von 1623, siehe Karin Paulsmeier, "Temporelationen bei Frescobaldi«, in: Alte Musik. Praxis und Reflexion, hrsg. von Peter Reidemeister und Veronika Gutmann, Winterthur 1983 , S. 197. Einen Sonderfall stellen die handschriftlichen Verrechnungen der "gantz« und »halb schlag « für Jacob Obrechts Missa Maria zart in dem Druck Concentus harmonici quattuor missarum ..., Basel: G. Mewes (1507?), RISM O 8, dar; vgl. Birgit Lodes, »An anderem Ort, auf andere Art: Petruccis und Mewes' Obrecht-Drucke «, in: Basler Jahrbuch für Historische Musikpraxis 25 (2001), S. 94 (Abb. 3). 
von Schülern schon früherer Zeiten mit der Mensuralnotation spiegeln. Da die Josquin-Messe 1561 sicher im Sinne von - und wohl auch aus dem Schülerkreis um - Glarean aufgeführt wurde, lässt sich die Frage nach Glareans Lehrmethode der Mensuralnotation stellen.

Glarean war sein Leben lang Lehrer und Erzieher, als Führer von Bursen in Basel (ab 1514) und später in Freiburg, als Erzieher in Paris (1517-1520), und als Universitätslehrer in Köln, Basel und ab 1529 in Freiburg. Die in diesem Zusammenhang maßgebliche Lehrschrift ist zunächst seine Isagoge in mvsicen (Basel 1516). ${ }^{12}$ Auf den nur zwanzig Seiten, die teilweise direkt in Glareans Dodekachordon übernommen wurden, ${ }^{13}$ findet sich nichts zur Mensuralnotation, da diese Schrift eine elementare Einführung in die "Musica" bietet und mehrstimmige Musik nicht berücksichtigt (ganz ähnlich wie die direkten Basler Vorläufer dieser Schulschrift von Balthasar Prasberg und Michael Keinspeck, aber anders als etwa Glareans offensichtliches Vorbild, Johannes Cochläus' Tetrachordum musices von 1512, der hierin weitergeht). Diese klare Beschränkung auf Musica plana und damit der Ausschluss von mensuriertem Gesang im Rahmen des elementaren Schulunterrichts findet sich bestätigt in Glareans Kommentar zur Freiburger Lateinschulordnung 1558. ${ }^{14}$ Hier fordert er: "Wann man in der schul die lateinisch Grammaticam vnd das Chorgesang wol lert vnd tribt, dunkt mich gnug sin ${ }^{15}$ Auch in seinen Detail-Empfehlungen zeigt sich eine Beschränkung auf die Vorbereitung des gottesdienstlichen Choralsingens. So sei am Freitagmittag »das ampt Sunntag ze singen, obersingen vnd die Responsorien an die tafel zeschriben, daselb die Knaben heisen abschriben vnd am Samstag den morgen behören doch for vom Cantor doch etlich mal ober solmisirt «. ${ }^{16}$ Andererseits ist aber sicher, dass Glarean in anderem Zusammenhang auch Mensuralmusik unterrichtete, belegt etwa durch die Unterweisung »im Figural-

12 Isagoge in musicen Henrici Glareani helvetii, Basel 1516 (eingesehen wurden die jeweils identischen Exemplare der Universitätsbibliothek Basel, Sign. kk II 24, DD V 4 Nr. 5 und C.H. II 19 Nr. 2). Zum Inhalt vgl. vor allem Bernhard Meier, »Heinrich Loriti Glareanus als Musiktheoretiker «, in: Beiträge zur Freiburger Wissenschafts- und Universitätsgeschichte 22 (1960), S. 65-112.

13 Ebda., S. 85.

14 Siehe Franz Bauer, „Ordnung der Freiburger Lateinschule von 1558, nebst den Gutachten des Glarean und Hartung «, in: Zeitschrift der Gesellschaft für Beförderung der Geschichts-, Alterthums- und Volkskunde von Freiburg, dem Breisgau und den angrenzenden Landschaften 1 (1867-1869), S. 79-104; Antonia Harter-Böhm, Zur Musikgeschichte der Stadt Freiburg im Breisgau um 1500, Freiburg/Brsg. 1968 (Veröffentlichungen aus dem Archiv der Stadt Freiburg im Breisgau, 10), S. 36-39.

15 F. Bauer, Ordnung, ebda., S. 93.

16 Ebda., S. 94 (hier »solinisirt« verlesen für »solmisiert«) und S. 96. 
gesange $\ll$ der drei Nonnen im Freiburger Franziskanerinnen-Kloster St. Clara (1556). ${ }^{17}$

In dem im Vorwort der Isagoge bereits indirekt angekündigten und bis in die späten 1530 er-Jahre ausgearbeiteten ${ }^{18}$ Dodekachordon äußert sich Glarean nur im dritten und letzten Buch zur Mensuralnotation, nachdem er im ersten Buch ausführlich den Gegenstand der Isagoge und im zweiten zentralen Buch seine Tonartenlehre darstellte. ${ }^{19}$ Die vergleichsweise kurzen Bemerkungen zur Musica figuralis (auf nur gut zwanzig Textseiten von insgesamt 43 Druckseiten, den übrigen Raum beanspruchen Musikbeispiele) haben - wie bereits Bernhard Meier konstatierte - vor allem einen Zweck: Glarean muss die Grundregeln der Mensuralnotation erklären, um die mehrstimmigen Musikbeispiele zur Exemplifizierung der Gültigkeit seiner Tonartenlehre zumindest im Prinzip lesbar zu machen. ${ }^{20}$

Glarean behandelt darin formal alle notwendigen Aspekte, vom Zeicheninventar (Noten-, Ligatur-, Pausen- und Mensurzeichen) über die Grundregeln der Augmentation, Diminution, Imperfektion, Alteration bis zu den Proportionen, folgend im Aufbau den Lehrbüchern von Cochläus oder Gaffurio. ${ }^{21}$ Schon bei einem Blick auf diese Grundlagen zeigen sich die konzeptuellen Schwächen seiner Darstellung im Sinne einer pragmatischen Anleitung, die letztlich auch die handschriftlichen Zusätze in der Josquin-Messe erklären können.

So beschreibt Glarean die Ligaturen nicht in ihrer rhythmischen Bedeutung, sondern nennt zunächst ihre Funktion bei der Textunterlegung (»Vt autem notulae uerborum syllabis cohaerent, aptarenturque, inuentae sunt notarum colligationes ...«, »Damit aber die Noten mit den Silben überein-

17 A. Harter-Böhm, Zur Muskgeschichte (wie Anm. 14), S. 45 f.

$18 \mathrm{H}$. Glarean, Isagoge (wie Anm. 12), Bg. Alv (in der Widmung an Peter Falk): »Sed licebit alias eadem sub incudem renouata, elimatiora reddere.« ( $(D e n n$ man wird mir gestatten, ein anderes Mal dasselbe Thema überarbeitet in abschließenderer Form wiederzugeben.«). Siehe auch Franz-Dieter Sauerborn, »Michael Rubellus von Rottweil als Lehrer von Glarean und anderen Humanisten«, in: Zeitschrift für Württembergische Landesgeschichte 54 (1995), S. 69-71.

19 Benutzt wurden neben dem Faksimile die Exemplare der Universitätsbibliothek Basel, Sign. kk I 1 und C.D. IV. 21. (Nr. 2), beide übrigens ohne Gebrauchsspuren.

20 B. Meier, Heinrich Loriti Glareanus (wie Anm. 12), S. 83. Dies erklärt auch, warum dem Dodekachordon der für dergleichen Titel wesentliche Abschnitt über den musikalischen Satz fehlt.

21 Dod., S. 196-239 (Kap. I-XII). Vgl. Tetrachordum Musices Joannis Coclei Norici, Nürnberg 1512, Reprint Hildesheim 1971, Tractatus quartus; Practica Mvsice Franchini Gafori Lavdensis, Mailand 1496, Reprint Farnborough 1967, Bologna 1972 und New York 1979, Liber secundus. 
stimmen und angepasst werden, hat man Verbindungen der Noten erfunden $){ }^{22}$ Es folgen acht Regeln, wobei Glarean hier fast wörtlich Cochläus, seinem Kölner Lehrer, folgt; dieser benötigte aber nur sieben, die zugefügte achte "Regula mit dem Hinweis auf Maxima-Ligaturen ist allerdings auch entbehrlich. Nun erscheinen diese acht Regeln aber nicht so abgefasst, dass man damit das Prinzip der Ligaturen in ihren rhythmischen Möglichkeiten verstehen könnte. So spricht Glarean meist im Singular von einer Einzelnote, obwohl Ligaturen per definitionem Mehrtongruppierungen sind und sich gerade aus der grafischen Abweichung gegenüber ihrer aus Neumen abgeleiteten Grundform die rhythmische Bedeutung am leichtesten erkennen lässt. So verfährt nämlich Gaffurio, dem Glarean an dieser Stelle aber wegen dessen angeblich >länglicher und umständlicher Beschreibung der Ligaturen nicht folgt. ${ }^{23}$ Zudem fehlt im Notenbeispiel des Dodekachordon die Auflösung der Ligaturen gemäß ihrem Wert als Einzelnoten. Dieses Manko ist erst in der Kurzfassung des Dodekachordon wettgemacht, dem 1557 auf lateinisch und 1559 in deutscher Übersetzung publizierten Auß Glareani Musick ein $v \beta z u g$, »denen zů hilff / so der Mathematick vnd vyllicht der Lateinschen sprach nitt gantz vnderricht «. ${ }^{24}$

Hier im deutschsprachigen Vßzug, der auf alles gelehrte Beiwerk verzichtet und mehrfach direkt die »leeriungen « als eigentliche Adressaten der Schrift anspricht, findet sich in der sechsten Ligatur-Regel auch das Zitat im Titel meines Beitrags: »Alle quadrat noten / so zů letst anhangend / sind alle lang. $\aleph^{25}$ Glarean meint damit das Ende »cum perfectione «ei absteigenden Ligaturen, was aber nur anhand des Notenbeispiels verständlich wird. Zudem ist die Kategorie »lang " nicht besonders differenziert, da bei einem System, das alles in Semibreven verrechnet, sowohl eine Brevis wie eine Longa "lang " sind.

Im Kapitel "De tactu siue cantandi mensura" des Dodekachordon beschreibt Glarean kurz die Möglichkeiten des Tempus perfectum cum prolatione maiori, »quae uel nunquam, uel rarissime usu uenit« (»was aber nie oder nur sehr selten vorkommt«), des Tempus imperfectum und Tempus perfec-

22 Dod., S. 197.

23 Ebda.: "Sed hoc loco nimis longus est magnifica diuisorum ambage Franchinus«. Vgl Gaffurio, Practica Mvsice (wie Anm. 21), Bg. aiiijv-avv .

24 Auß Glareani Musick ein $p \beta z u g$ / mit verwilligung vnd hilff Glareani ..., Basel 1559, Reprint Leipzig 1975, S. xcix-cij; Zitat auf dem Titelblatt. Inhaltlich identisch ist Mvsicae Epitome ex Glareani Dodecachordo ..., Basel 1559 (eingesehen wurde das Exemplar der Universitätsbibliothek Basel, Sign. Rb73), von dem es auch eine erste Ausgabe von 1557 geben soll.

25 Auß Glareani Musick ein $v \beta z u g$, ebda., S. cj. 
tum, »quae usu maxime recepta est« (»welche vor allem gebraucht werden «). ${ }^{26}$ Bemerkenswert ist nun die fast lapidare Reduktion oder sogar Simplifizierung im V $\beta z u g$ : "De tactu« ist hier mit "Von schlag « übersetzt und gleich im ersten Satz der Wechsel der »schlag«-Einheit von der Brevis auf die Semibrevis beschrieben - mit dem bezeichnenden pragmatischen Kommentar »ist auch leichterer zůlernen ${ }^{27}{ }^{27}$ Im Folgenden werden im Vßzug dann auch alle Notendauern in einzelne Semibreven bzw. "schlege u umgerechnet - wie sie sich in den handschriftlichen Annotationen des Antico-Drucks finden.

Es scheint wenig wahrscheinlich, dass aufgrund der bei Glarean gegebenen Erläuterungen - obwohl theoretisch korrekt - die Ligaturen in ihrer rhythmischen Bedeutung wirklich verstanden werden können. Vermutlich wurde dieses Thema vor allem in eine mündliche Unterweisung delegiert, wie sie Glarean zum Problem der Augmentation und Diminution empfiehlt: "Quare quod ad augmentationem attinet, ad cantores lectorem remittimus (»weshalb wir bezüglich der Augmentation die Leser an die Cantoren verweisen «). ${ }^{28}$ Und in der mündlichen Unterweisung wurden diese Fragen dann wohl auf pragmatische Antworten reduziert, wie es im konkreten Fall szu machen ist

M.D.LXI, in natali uirginis matris, hanc Missam Friburgi in summo templo in D. Glareani gratiam dexterrime demodularj sumus.

Warum Kollegen, Schüler oder Freunde von Glarean 1561 gerade diese Messe aussuchten, um ihn mit einer Aufführung im Freiburger Münster zu ehren, ${ }^{29}$ ist nicht schwer zu beantworten, obwohl eine Aufführung von etwa fünfzig Jahre alter Musik (zudem aus einem gleichermaßen alten Druck) durchaus bemerkenswert ist: ${ }^{30}$ Glarean war bekanntlich nicht nur ein besonderer Verehrer von Josquin, er selbst lobt im Dodekachordon gerade diese

26 Dod., S. 203-205 (Kap. VII).

27 Auß Glareani Musick ein v $\beta z u g$ (wie Anm. 24), S. cix-cxj (falsch paginiert); Zitate S. cix.

28 Dod., S. 205.

29 "Friburgi in summo templo« wird sich aufgrund der biografischen Situation sehr wahrscheinlich auf Freiburg im Breisgau beziehen, auch die Aufführung einer Marien-Messe in dem Unserer Lieben Frauen geweihten Freiburger Münster ist passend.

$30 \mathrm{Vgl}$. etwa die als selten eingeschätzte, fast gleichzeitige (1559/60) Josquin-Rezeption durch den Regensburger Cantor Johannes Buechmeier; siehe James Haar, "Josquin as Interpreted by a Mid-Sixteenth-Century German Musician«, in: Festschrift fiur Horst Leuchtmann zum 65. Geburtstag, hrsg. von Stephan Hörner und Bernhold Schmid, Tutzing 1993, S. 179-205. 
Messe und widmet in einem "Iusquini Pratensis encomium " betitelten Kapitel sogar einen eigenen Abschnitt nur dem Gloria der Messe. ${ }^{31}$ Vordergründig geht es ihm um die seltene, aber auch selten so gelungene Verbindung des Mixolydischen mit seinem plagalen Modus, die Josquin - und hierin Brumel übertreffend - anwandte, "nec inueniri meliorem cantum posse" (»dass ein besserer Gesang nicht erfunden werden könne"). Weiter schreibt Glarean, dass diese hohe Meinung durch Ioannes Vannius (alias Johannes Wannenmacher) bestätigt würde, der mehr von der Sache verstehe. Besonders im ersten Teil des Glorias zu dem Textwort "Primogenitus" sei der "größte Affekt« ausgedrückt, andere würden dem zweiten Teil den Vorzug geben, aber - schließt Glarean rhetorisch - es gebe keinen Abschnitt darin, der nichts habe, was man nicht anders als bewundern könne (»Maximus eius affectus mihi quidem expressus uidetur in uerbo Primogenitus, prima huius cantionis parte. Alij secundam partem preferunt, sed omnino nulla eius pars est, quae non habeat, quod plurimum mireris«).

Es gibt aber noch eine weitere Aussage Glareans zu dieser Messe von Josquin, die bislang offenbar unbeachtet blieb. Auf den eingangs genannten Seiten im Antico-Druck mit dem Beginn der Josquin-Messe steht links oben eine handschriftliche griechische Textzeile (siehe Abbildung 6):

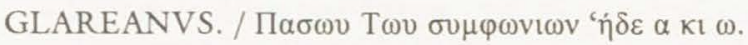

Glarean: Von allen »Symphonien« [im Sinne von mehrstimmigen Kompositionen] ist diese das $\mathrm{A}$ und $\mathrm{O}$ [im Sinne von die allerbeste].

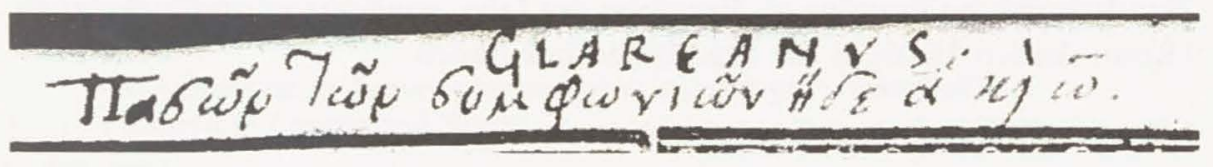

Abbildung 6: wie Abbildung 1, fol. 114 (Ausschnitt)

Tatsächlich handelt es sich bei diesem Vermerk um ein direktes Zitat Glareans. Es findet sich im Dodekachordon unten auf der Seite mit dem Beginn des Notenbeispiels mit dem Gloria aus Josquins Messe (siehe Abbildung 7). ${ }^{32}$

31 Dod., Buch III, Kap. XXIIII (S. 362-429), zum »Et in terra pax«S. 365f. (mit Notenteil S. 392-401); Zitate S. 366. Am Rande sei angemerkt, dass aufgrund der Lesarten und Ligaturensetzung offensichtlich nicht Petruccis Druck von 1514, sondern eine Version ähnlich der in Anticos Druck von 1516 als Vorlage für die Beispiele im Dodekachordon diente.

32 Dod., S. 393 (dies wurde bislang sowohl in den Übersetzungen des Dodekachordon von Peter Bohn und Clement A. Miller wie auch in den Editionen der Josquin-Messe von Albert Smijers und von Willem Elders im Rahmen der New Josquin Edition, Bd. 3: Masses based on gregorian Chant, Tl. 1, Utrecht 2003, übersehen). 


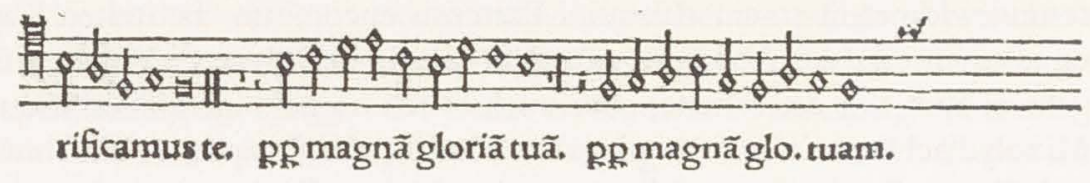

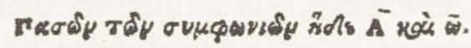

\section{Refiduum}

Abbildung 7: Dod., S. 393 (Ausschnitt)

Dieser Kommentar unterstreicht die besondere Bedeutung und Wertschätzung gerade dieser Musik für Glarean und macht verständlich, warum Josquins Missa de beata virgine für eine feierliche Ehrung Glareans ausgewählt wurde. Offen bleibt aber die Frage, warum die musikalische Ehrung gerade 1561 stattfand, da in dieses Jahr kein erkennbares biografisch markantes Jubiläum fällt. Es kann aber spekuliert werden, ob vielleicht der allmähliche Rückzug des alten und auch kränkelnden Glarean von seinen Unterrichtspflichten an der Freiburger Universität ab dem Wintersemester 1560 den Anlass dazu gab. ${ }^{33}$

Anhang

Handschriftliche Einträge im Exemplar des Liber qvindecim missarvm electarvm (Rom: Antico 1516) in der Stadtbibliothek Baden

in Josquin Desprez, Missa de beata virgine

fol. cxivv:

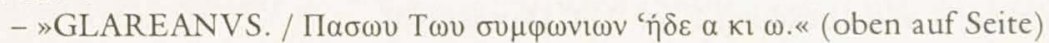

- Ziffern über Ligaturen im Christe bei Cantus und Tenor (je »2« für Kennzeichnung der Brevis am Ende von Ligaturen cum opposita proprietate)

- »Tenor (marginal)

fol. cxv:

- Ziffern über Ligaturen im Christe bei Altus und Bassus (je »2« für Kennzeichnung der Brevis am Ende von Ligaturen cum opposita proprietate)

- $\gg$ M.D.LXI, in natali uirginis matris, hanc Missam Friburgi in summo

templo in D. Glareani gratiam dexterrime demodularj sumus.« (zwischen Altus und Bassus)

33 Siehe Otto Fridolin Fritzsche, Glarean. Sein Leben und seine Schriften, Frauenfeld 1890, S. $78 \mathrm{f}$. 
fol. $\mathrm{cxv}^{\mathrm{v}}$ :

- »Altus (marginal)

fol. $\operatorname{cxix}^{\mathrm{v}}$ :

- Ziffer »6 6 über Pause und » 3 « über Einzelnote im Cantus; $» 12$ « und » 9 « über Pausen im Tenor, " 2 « über Brevis

fol. cxx:

- » 3 « über Pause, » 3 « über Brevis im Altus; mehrere Ziffern im Bassus

fol. $\mathrm{cxx}^{\mathrm{v}}$;

- »Altus« (marginal)

fol. cxxi:

- Tenor $\ll$ (marginal)

fol. cxxiv :

- »Altus« (marginal)

fol. cxxii:

- »Tenor« (marginal)

fol. cxxiiiv :

- "Tenor. Nach XII schlegenn fahrt V vox an ein quint über dem Tenor.» (marginal)

- Ziffern im Tenor

fol. cxxiiii:

- $\gg$ Altus« (marginal)

- Ziffern in Altus und Bassus

- Akzidens b im Altus (T. 10,2)

fol. cxxiiii" :

- »Tenor / Quinta vox gath uß dem Tenor nach acht schlegenn in obere quint." (marginal)

fol. cxxvi":

- »Quinta uox ex Tenore post tempora in $\Delta 1 \alpha \pi \varepsilon v \tau \varepsilon \ll$ (marginal)

- Ziffern in Cantus und Tenor

fol. cxxvii:

- Ziffern in Altus und Bassus

fol. cxxviii:

- Ziffer $» 8 \ll$ im Altus zu Beginn

fol. cxxviiii":

- »Quinta uox ex Tenore post octo pausas in $\Delta t \alpha \pi \varepsilon v \tau \varepsilon \ll$ (marginal)

im übrigen Band

Titelblatt:

- »LEO X. PONT. MAX. / 1.5.6.1.« (oberhalb des Papstbildes) - dies von der derselben Hand wie die Einträge in der Josquin-Messe

fol. xxv:

- Bleistift-Einträge in freie Notenlinien - Hand aus dem 19. Jahrhundert

fol. lxxi, fol. Ixxvii und fol. lxxivv:

- Ergänzung eines fehlenden Signum congruentiae (vielleicht vom Drucker) 
weitere (und ähnliche Vermerke wie in Josquins Missa de beata virgine) in Josquins Missa ad fugam:

fol. cxxix ${ }^{v}$ : Ziffer

fol. cxxxviii: $\downarrow$ für Auflösung des b-Akzidens

fol. cxl ${ }^{v}$ : Textänderung im Agnus Dei (statt »dona nobis pacem« nun »miserere nobis $\ll)$

sowie in Brumels Missa pro defunctis

fol. clvi: Ziffern (jeweils von derselben Hand wie die Einträge in der JosquinMesse)

eingeklebt im hinteren Deckel: oblonger Zettel mit einer Regel für die Imperfectio ad partem

Text: "Regula de Imperfectione partiali / Si in tempore perfecto Longam notulam unica tantum / semibreuis aut pausa sequitur, Imperfectio non Longae notulae, sed altri breui, quarum duos illa continet, ascribatur." (Handschrift des 16. Jahrhunderts) 